\title{
Local Structures and Dynamics of Imidazole Molecules in Poly(vinylphosphonic acid)-Imidazole Composite Investigated by Molecular Dynamics
}

Yuta Hori, ${ }^{* 1}$ Toshiya Suetake, ${ }^{2}$ Yoshihito Shiota,${ }^{3}$ Kazunari Yoshizawa, ${ }^{3}$ Yasuteru Shigeta, ${ }^{1}$ Tomonori Ida, ${ }^{2}$ and Motohiro Mizuno ${ }^{2,4}$

${ }^{1}$ Center for Computational Sciences, University of Tsukuba, Tsukuba, 305-8577 Japan.

${ }^{2}$ Chemistry Course, Division of Material Chemistry, Graduate School of Natural Science and Technology, Kanazawa University, Kanazawa 920-1192 Japan.

${ }^{3}$ Institute for Materials Chemistry and Engineering and IRCCS, Kyushu University, Fukuoka 819-0395 Japan.

${ }^{4}$ Nanomaterials Research Institute (NanoMaRI), Kanazawa University, Kanazawa 920-1192 Japan.

*E-mail: hori@ccs.tsukuba.ac.jp 


\section{Table of Contents:}

Figure S1. A snapshot of the MD simulations of pure Im.

Figure S2. Three types of local structures of Im dimer extracted from Fig. S1: (a) linear hydrogen-bond dimer, (b) $\pi$-stacked dimer, and (c) T-shaped dimer geometries.

Figure S3. Two types of hydrogen-bond structures of phosphonic acid group and imidazolium extracted from a snapshot of the molecular dynamics simulations of PVPA-1Im.

Figure S4. Computed interaction energies (a) between Im molecules and (b) between the PA group and $\mathrm{ImH}^{+}$by using DFT calculations at the CAM-B3LYP/aug-cc-pVDZ level of theory for previous optimized structures. ${ }^{32}$

Figure S5. A snapshot of the MD simulations of PVPA-2Im.

Figure S6. A snapshot of the MD simulations of PVPA-3Im.

Figure S7. Rotational autocorrelation functions of $\operatorname{Im}\left(C_{\operatorname{Im}}(\mathrm{t})\right.$ and $\left.\ln C_{\operatorname{Im}}(\mathrm{t})\right)$ in pure $\operatorname{Im}$.

Figure S8. Rotational autocorrelation functions of $\operatorname{ImH}^{+}\left(C_{\mathrm{ImH}}(\mathrm{t})\right.$ and $\left.\ln C_{\mathrm{ImH}+}(\mathrm{t})\right)$ in PVPA1 Im.

Figure S9. Rotational autocorrelation functions of $\operatorname{ImH}^{+}\left(C_{\mathrm{ImH}+}(\mathrm{t})\right.$ and $\left.\ln C_{\mathrm{ImH}+}(\mathrm{t})\right)$ in PVPA2Im.

Figure S10. Rotational autocorrelation functions of $\operatorname{Im}\left(C_{\operatorname{Im}}(\mathrm{t})\right.$ and $\left.\ln C_{\operatorname{Im}}(\mathrm{t})\right)$ in PVPA-2Im.

Figure S11. Rotational autocorrelation functions of $\operatorname{ImH}^{+}\left(C_{\operatorname{ImH}+}(\mathrm{t})\right.$ and $\left.\ln C_{\operatorname{ImH}+}(\mathrm{t})\right)$ in PVPA3Im.

Figure S12. Rotational autocorrelation functions of $\operatorname{Im}\left(C_{\operatorname{Im}}(\mathrm{t})\right.$ and $\left.\ln C_{\operatorname{Im}}(\mathrm{t})\right)$ in PVPA-3Im.

Figure S13. RDF of N2-N3 in PVPA-2Im and PVPA-3Im.

Table S1. Force field parameters for PVPA oligomer with two repeat units. 
Table S2. Force field parameters for $\mathrm{Im}$ and $\mathrm{ImH}^{+}$. 


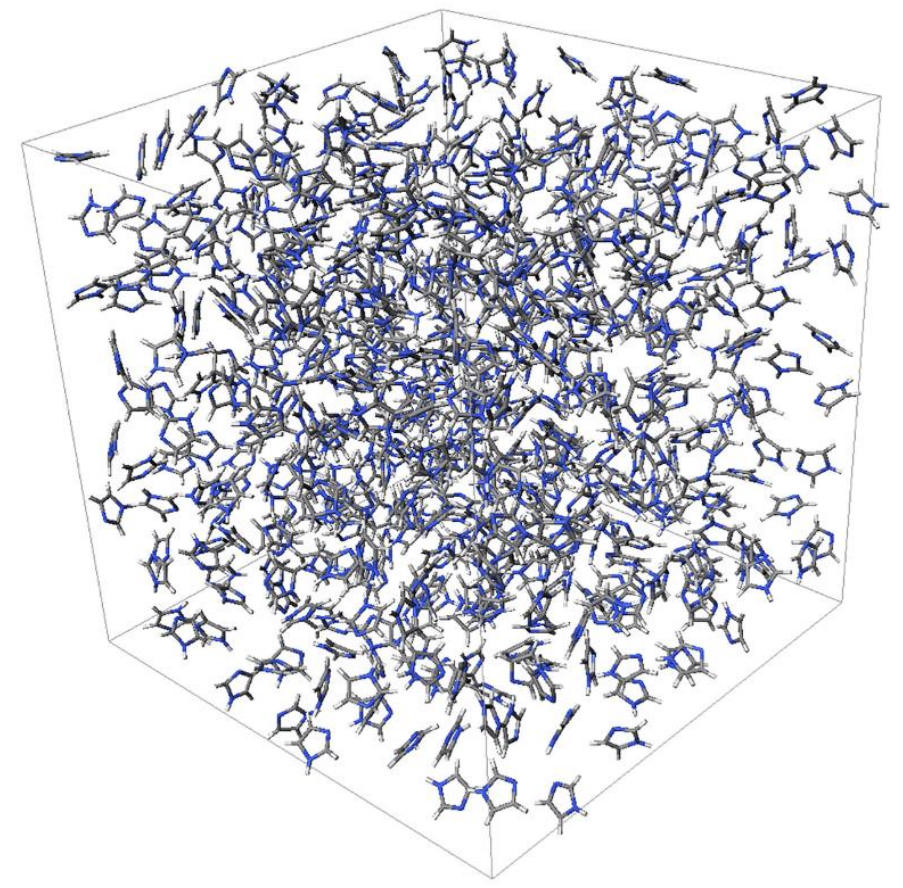

Figure S1. A snapshot of the MD simulations of pure Im.

(a)

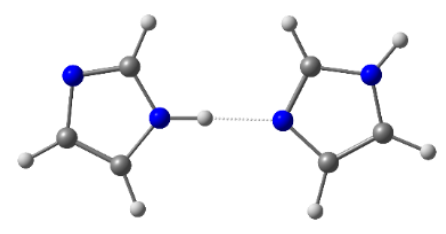

(b)

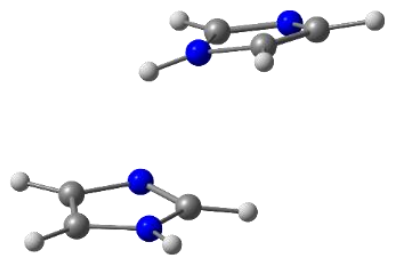

(c)

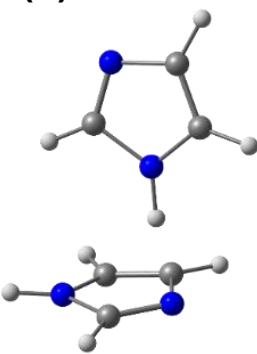

Figure S2. Three types of local structures of Im dimer extracted from Fig. S1: (a) linear hydrogen-bond dimer, (b) $\pi$-stacked dimer, and (c) T-shaped dimer geometries. 
(a)

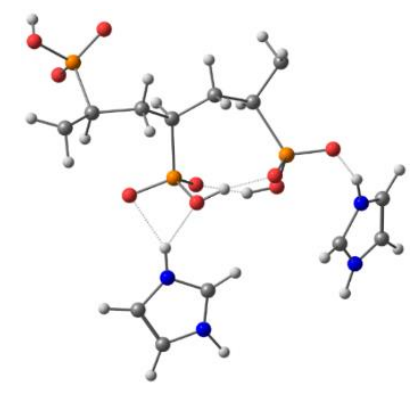

(b)

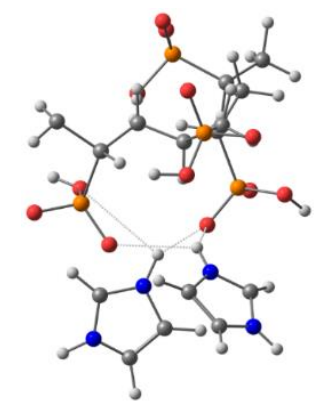

Figure S3. Two types of hydrogen-bond structures of phosphonic acid group and imidazolium extracted from a snapshot of the molecular dynamics simulations of PVPA-1Im.

(a)

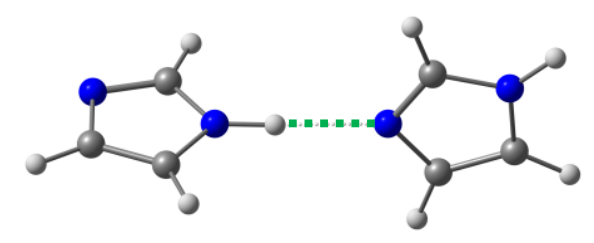

9.27

(b)

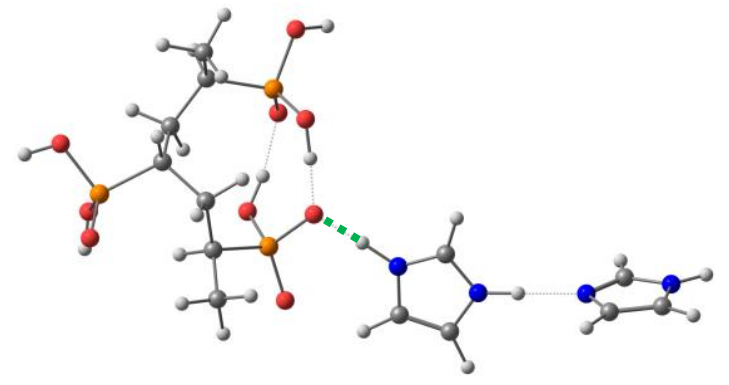

95.8

(kcal $/ \mathrm{mol})$

Figure S4. Computed interaction energies (a) between Im molecules and (b) between the PA group and $\mathrm{ImH}^{+}$by using DFT calculations at the CAM-B3LYP/aug-cc-pVDZ level of theory for previous optimized structures. ${ }^{32}$ 


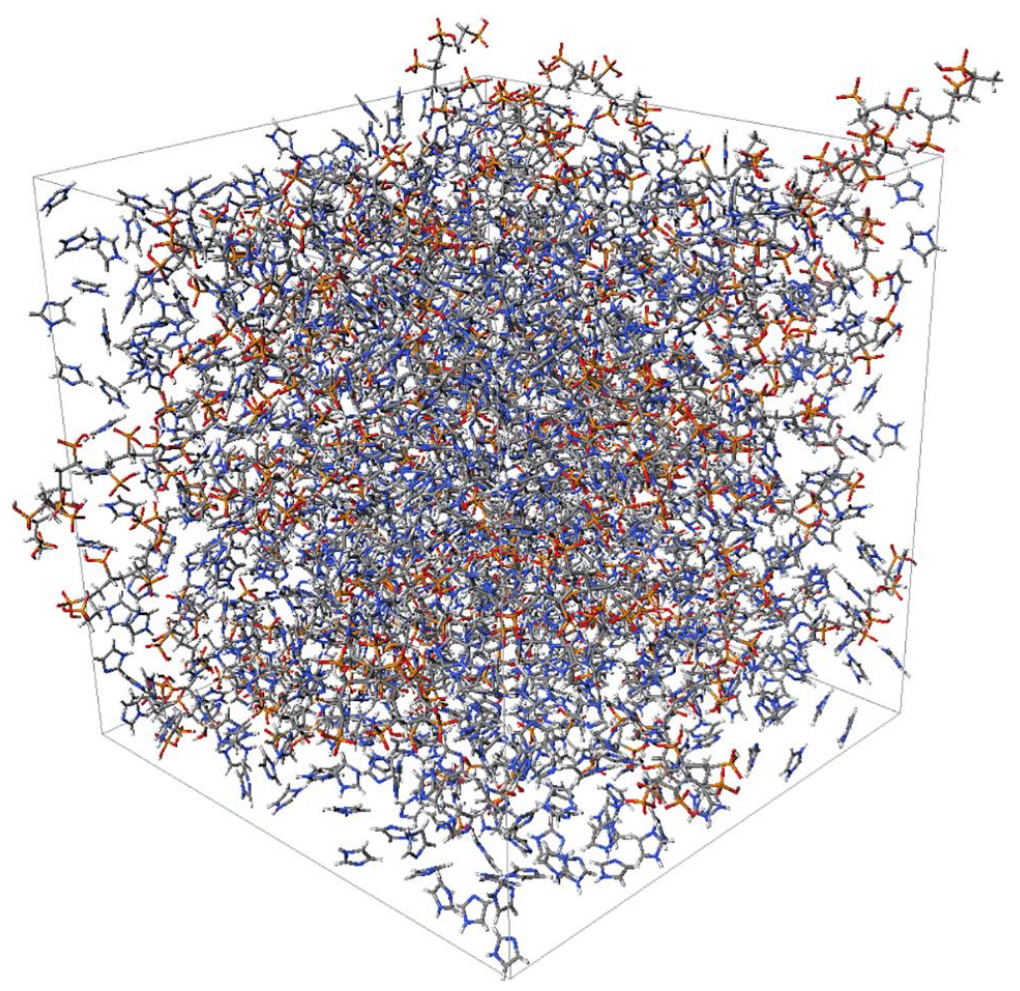

Figure S5. A snapshot of the MD simulations of PVPA-2Im. 


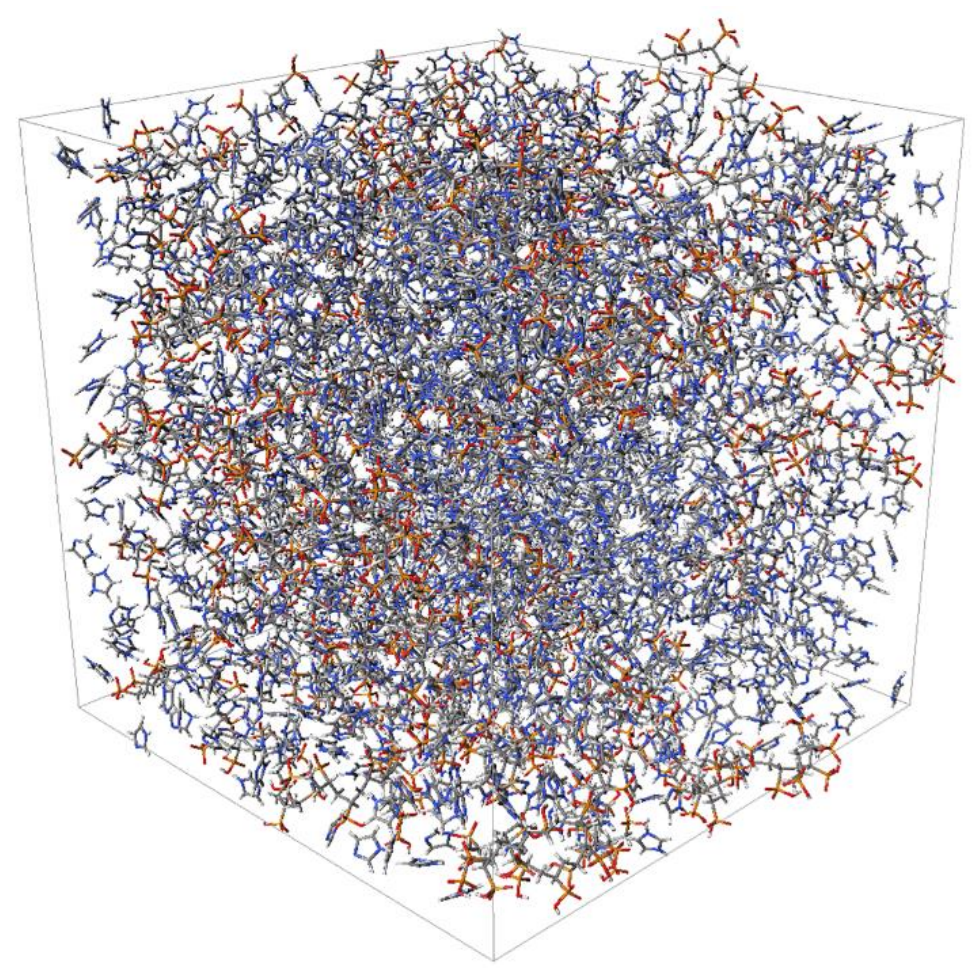

Figure S6. A snapshot of the MD simulations of PVPA-3Im.
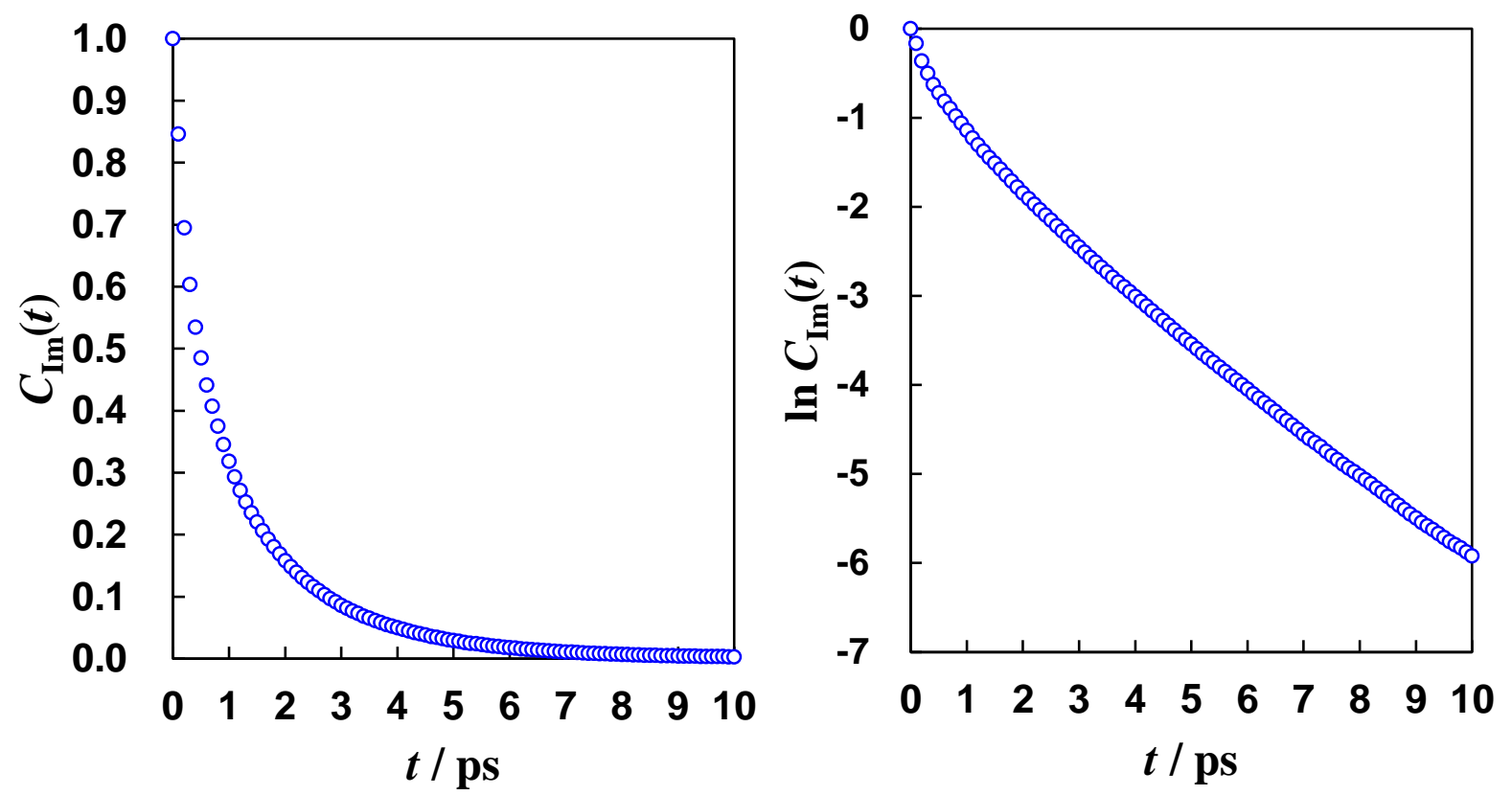

Figure S7. Rotational autocorrelation functions of $\operatorname{Im}\left(C_{\operatorname{Im}}(\mathrm{t})\right.$ and $\left.\ln C_{\operatorname{Im}}(\mathrm{t})\right)$ in pure $\operatorname{Im}$. 

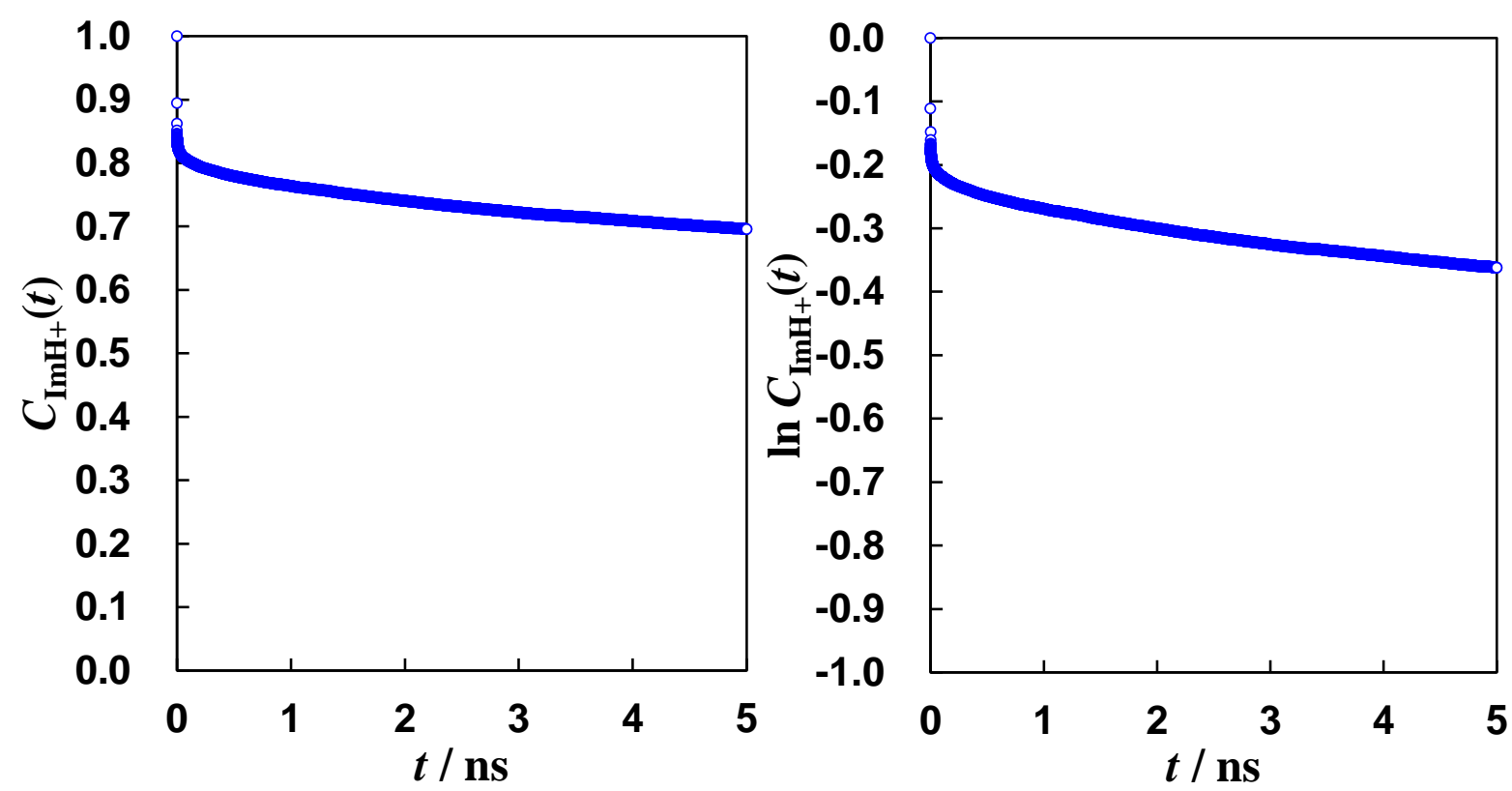

Figure S8. Rotational autocorrelation functions of $\operatorname{ImH}^{+}\left(C_{\mathrm{ImH}+}(\mathrm{t})\right.$ and $\left.\ln C_{\mathrm{ImH}+}(\mathrm{t})\right)$ in PVPA$1 \mathrm{Im}$.
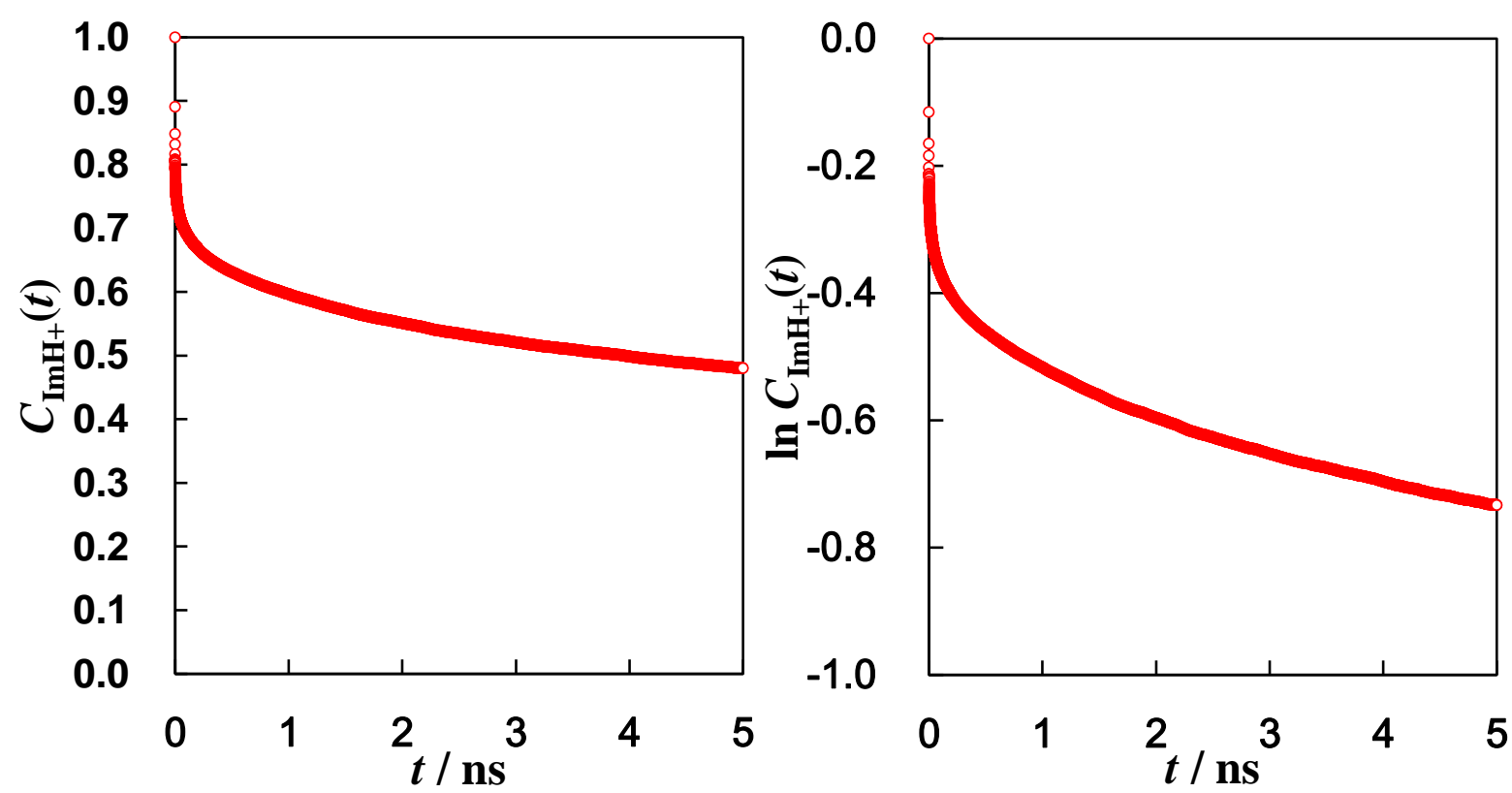

Figure S9. Rotational autocorrelation functions of $\operatorname{ImH}^{+}\left(C_{\mathrm{ImH}+}(\mathrm{t})\right.$ and $\left.\ln C_{\mathrm{ImH}+}(\mathrm{t})\right)$ in PVPA2Im. 

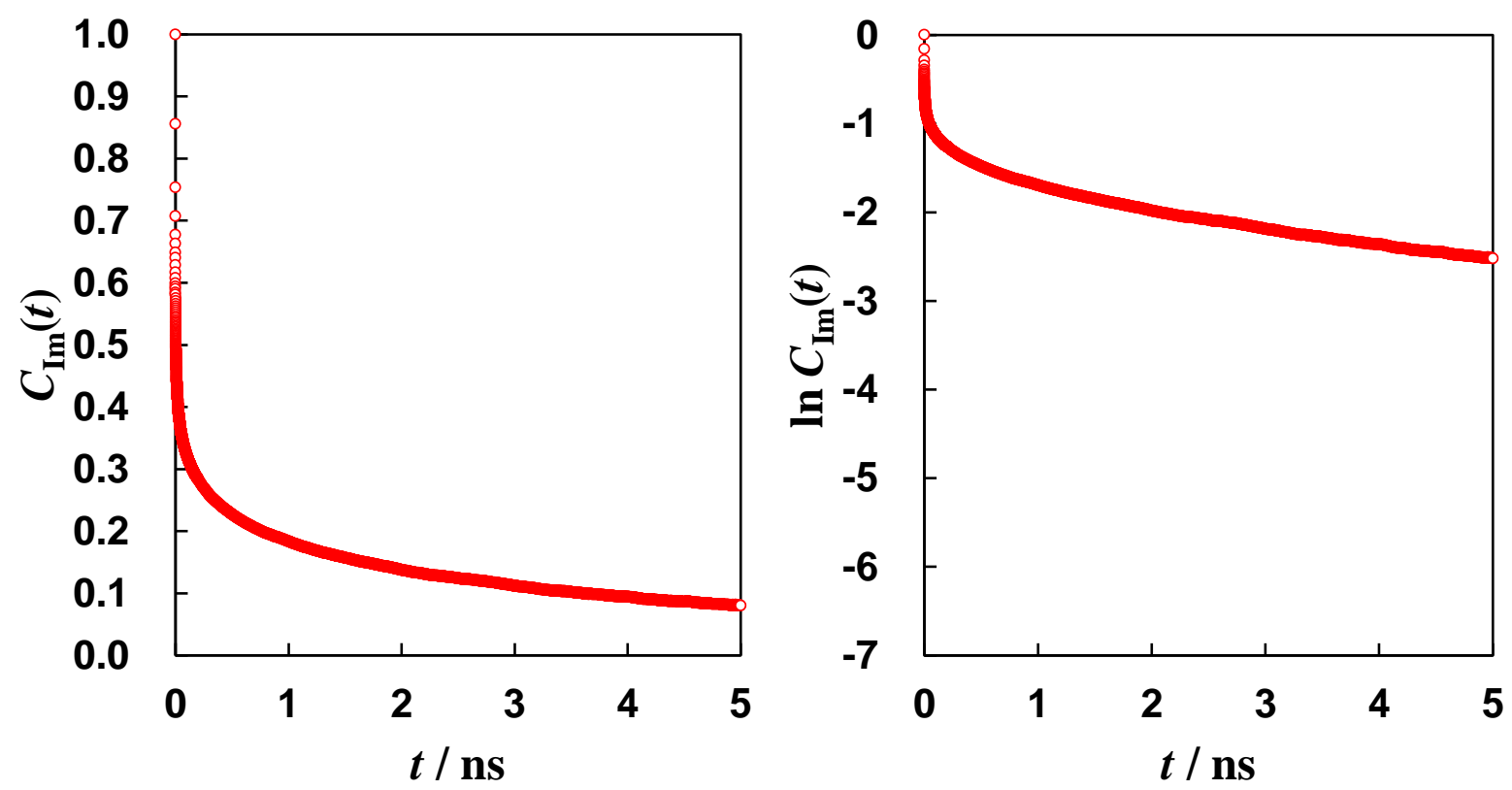

Figure S10. Rotational autocorrelation functions of $\operatorname{Im}\left(C_{\operatorname{Im}}(\mathrm{t})\right.$ and $\left.\ln C_{\operatorname{Im}}(\mathrm{t})\right)$ in PVPA-2Im.
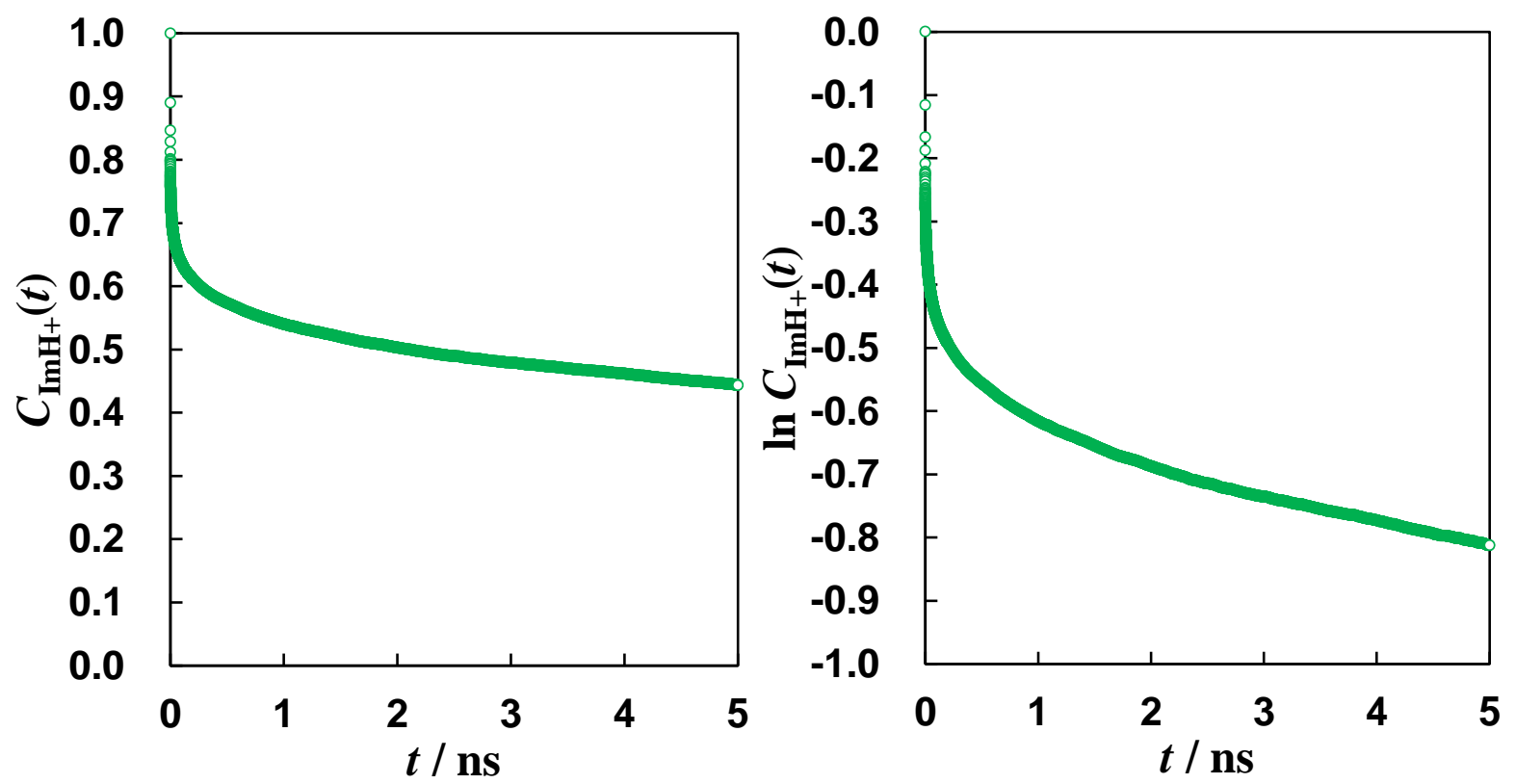

Figure S11. Rotational autocorrelation functions of $\operatorname{ImH}^{+}\left(C_{\operatorname{ImH}+}(\mathrm{t})\right.$ and $\left.\ln C_{\operatorname{ImH}+}(\mathrm{t})\right)$ in PVPA3Im. 

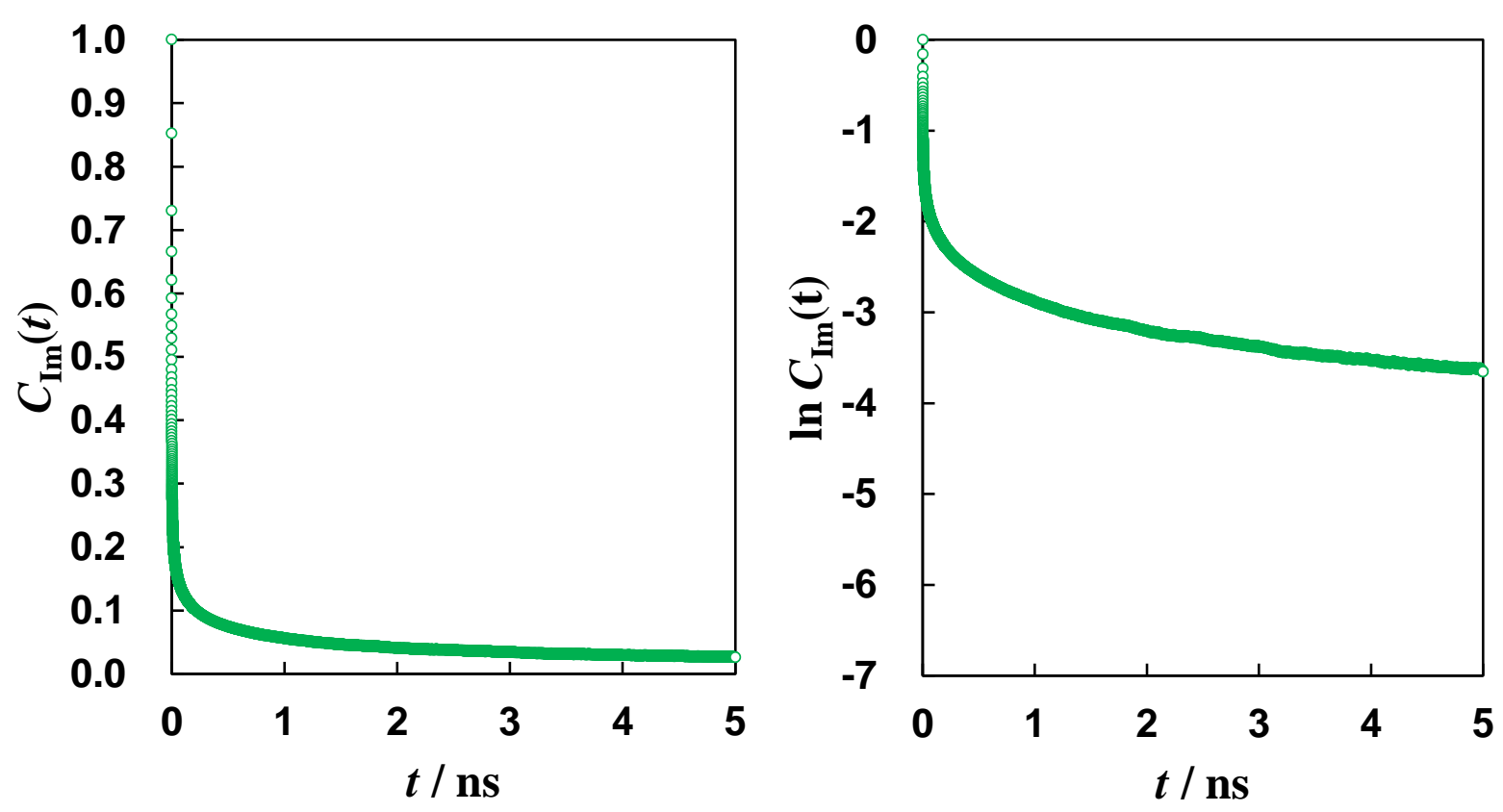

Figure S12. Rotational autocorrelation functions of $\operatorname{Im}\left(C_{\operatorname{Im}}(\mathrm{t})\right.$ and $\left.\ln C_{\operatorname{Im}}(\mathrm{t})\right)$ in PVPA-3Im.

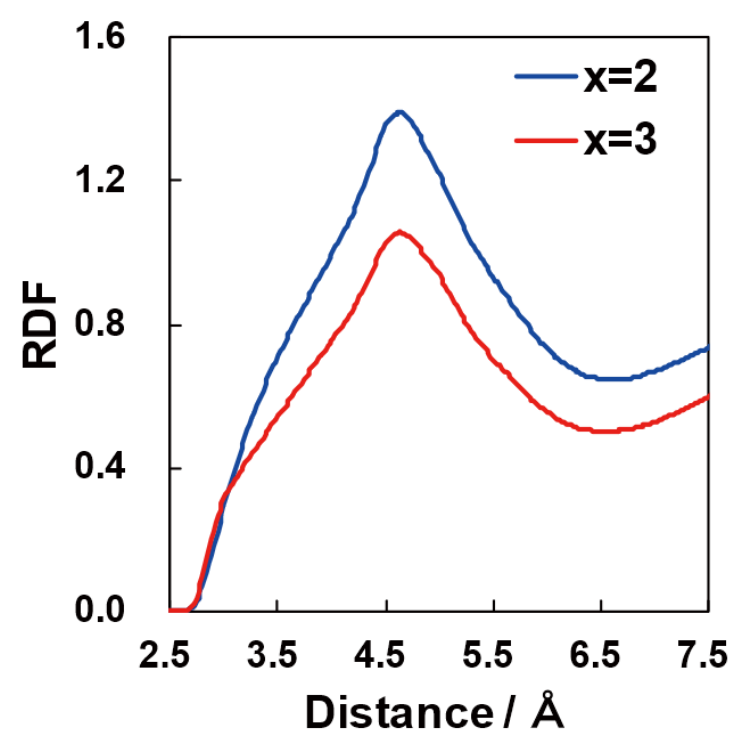

Figure S13. RDF of N2-N3 in PVPA-2Im and PVPA-3Im. 
Table S1. Force field parameters for PVPA oligomer with two repeat units.

\begin{tabular}{|c|c|c|c|}
\hline \multicolumn{4}{|c|}{$E_{\mathrm{bond}}\left(r_{i j}\right)=\frac{1}{2} K_{b}\left(r_{i j}-r_{0}\right)^{2}$} \\
\hline$E_{\text {bond }}$ & $r_{0}(\mathrm{~nm})$ & & $K_{b}\left(\mathrm{~kJ} / \mathrm{mol} / \mathrm{nm}^{2}\right)$ \\
\hline p5-o & 0.14870 & & 443,090 \\
\hline p5-oh & 0.16150 & & 289,530 \\
\hline p5-c3 & 0.18390 & & 148,280 \\
\hline oh-ho & 0.09730 & & 471,540 \\
\hline $\mathrm{c} 3-\mathrm{c} 3$ & 0.15380 & & 194,560 \\
\hline c3-hc & 0.10970 & & 314,550 \\
\hline \multicolumn{4}{|c|}{$E_{\text {angle }}\left(\theta_{i j k}\right)=\frac{1}{2} K_{\theta}\left(\theta_{i j k}-\theta_{0}\right)^{2}$} \\
\hline$E_{\text {angle }}$ & $\theta$ (deg) & & $K_{\theta}\left(\mathrm{kJ} / \mathrm{mol} / \mathrm{rad}^{2}\right)$ \\
\hline p5-oh-ho & 110 & & 494 \\
\hline p5-c3-hc & 108 & & 457 \\
\hline p5-c3-c3 & 112 & & 674 \\
\hline $\mathrm{o}-\mathrm{p} 5-\mathrm{o}$ & 116 & & 715 \\
\hline o-p5-oh & 115 & & 685 \\
\hline $\mathrm{o}-\mathrm{p} 5-\mathrm{c} 3$ & 113 & & 506 \\
\hline oh-p5-c3 & 103 & & 518 \\
\hline $\mathrm{c} 3-\mathrm{c} 3-\mathrm{c} 3$ & 112 & & 543 \\
\hline $\mathrm{c} 3-\mathrm{c} 3-\mathrm{hc}$ & 110 & & 392 \\
\hline hc-c3-hc & 108 & & 326 \\
\hline \multicolumn{4}{|c|}{$E_{\text {torsion }}\left(\phi_{i j k l}\right)=k_{\phi}\left\{1+\cos \left(n \phi_{i j k l}-\phi_{s}\right)\right\}$} \\
\hline$E_{\text {torsion }}$ & $\phi_{s}(\operatorname{deg})$ & $n$ & $k_{\phi}(\mathrm{kJ} / \mathrm{mol})$ \\
\hline $\mathrm{p} 5-\mathrm{c} 3-\mathrm{c} 3-\mathrm{c} 3$ & 0 & 3 & 0.65084 \\
\hline p5-c3-c3-hc & 0 & 3 & 0.65084 \\
\hline o-p5-oh-ho & 0 & 3 & 1.53553 \\
\hline
\end{tabular}




\begin{tabular}{|c|c|c|c|}
\hline $\mathrm{o}-\mathrm{p} 5-\mathrm{c} 3-\mathrm{c} 3$ & 0 & 3 & 0.09298 \\
\hline $\mathrm{o}-\mathrm{p} 5-\mathrm{c} 3-\mathrm{hc}$ & 0 & 3 & 0.09298 \\
\hline oh-p5-c3-c3 & 0 & 3 & 0.09298 \\
\hline $\mathrm{oh}-\mathrm{p} 5-\mathrm{c} 3-\mathrm{hc}$ & 0 & 3 & 0.09298 \\
\hline ho-oh-p5-c3 & 0 & 3 & 2.23147 \\
\hline$c 3-c 3-c 3-c 3$ & 0 & 3 & 0.54392 \\
\hline$c 3-c 3-c 3-c 3$ & 180 & 2 & 1.21336 \\
\hline$c 3-c 3-c 3-c 3$ & 0 & 1 & 0.46024 \\
\hline c3-c3-c3-hc & 0 & 3 & 0.33472 \\
\hline hc-c3-c3-hc & 0 & 3 & 0.50208 \\
\hline \multicolumn{4}{|c|}{$\left(\frac{\sigma_{i j}}{r_{i j}}\right)^{12}-\left(\frac{\sigma_{i j}}{r_{i j}}\right)^{6}$} \\
\hline$E_{\mathrm{LJ}}$ & \multicolumn{2}{|c|}{$\varepsilon_{i i}(\mathrm{~kJ} / \mathrm{mol})$} & $\sigma_{i i}(\mathrm{~nm})$ \\
\hline p5 & \multicolumn{2}{|c|}{0.9602280} & 0.3694020 \\
\hline o & \multicolumn{2}{|c|}{0.6121190} & 0.3048120 \\
\hline oh & \multicolumn{2}{|c|}{0.3891120} & 0.3242870 \\
\hline $\mathrm{c} 3$ & \multicolumn{2}{|c|}{0.4510350} & 0.3397710 \\
\hline ho & \multicolumn{2}{|c|}{0.0196648} & 0.0537925 \\
\hline hc & \multicolumn{2}{|c|}{0.0870272} & 0.2600180 \\
\hline
\end{tabular}

Table S2. Force field parameters for $\mathrm{Im}$ and $\mathrm{ImH}^{+}$.

\begin{tabular}{ccc}
\hline & $E_{\text {bond }}\left(r_{i j}\right)=\frac{1}{2} K_{b}\left(r_{i j}-r_{0}\right)^{2}$ \\
\hline$E_{\text {bond }}$ & $r_{0}(\mathrm{~nm})$ & $K_{b}\left(\mathrm{~kJ} / \mathrm{mol} / \mathrm{nm}^{2}\right)$ \\
\hline cc-na & 0.13800 & 296,650 \\
cd-na & 0.13800 & 296,650
\end{tabular}




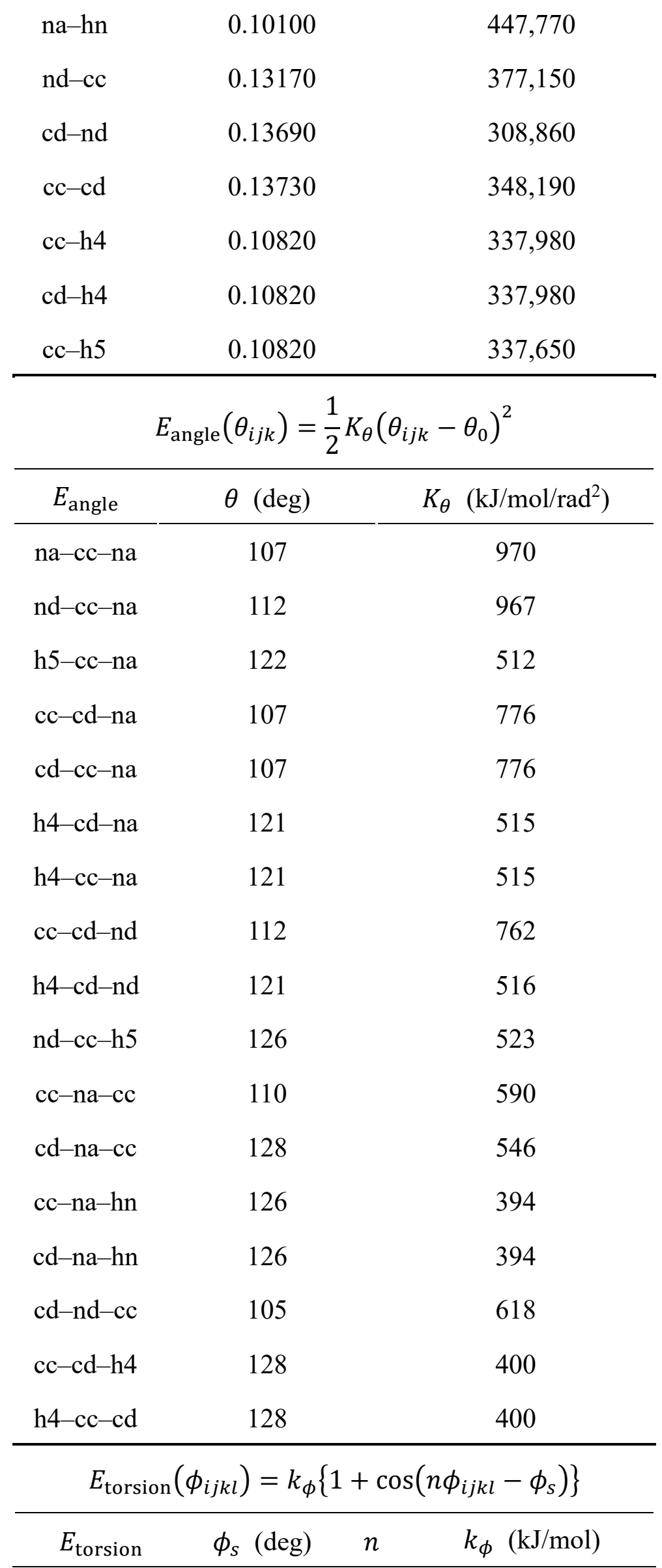




\begin{tabular}{llll}
\hline na-cc-cd-na & 180 & 2 & 16.736 \\
na-cc-cd-nd & 180 & 2 & 16.736 \\
h4-cc-cd-na & 180 & 2 & 16.736 \\
na-cc-cd-h4 & 180 & 2 & 16.736 \\
cd-nd-cc-na & 180 & 2 & 19.874 \\
cd-nd-cc-h5 & 180 & 2 & 19.874 \\
cc-na-cc-na & 180 & 2 & 7.1128 \\
cd-na-cc-na & 180 & 2 & 7.1128 \\
cd-na-cc-h5 & 180 & 2 & 7.1128 \\
hn-na-cc-na & 180 & 2 & 7.1128 \\
cc-h4-cd-na & 180 & 2 & 4.6024 \\
na-cc-h4-cd & 180 & 2 & 4.6024 \\
h5-na-cc-na & 180 & 2 & 4.6024 \\
h5-na-cc-nd & 180 & 2 & 4.6024 \\
c4-cc-cd-nd & 180 & 2 & 16.736 \\
cc-cd-na-hn & 180 & 2 & 16.736 \\
h4-cc-cd-h4 & 180 & 2 & 7.1128 \\
cc-na-cc-nd & 180 & 2 & 7.1128 \\
nd-cc-na-hn & 180 & 2 & 7.116024 \\
cc-h4-cd-nd & 180 & 2 & 7.1128 \\
cc-cd-nd-cc & 180 & 2 & 1.1128 \\
h4-cd-nd-cc-na-cc-na-na-cc & 180 & 2 & 2 \\
\hline
\end{tabular}




\begin{tabular}{cccc} 
cc-cd-na-hn & \multicolumn{1}{c}{180} & 2 & 4.6024 \\
h5-cc-na-hn & 180 & 2 & 7.1128 \\
h4-cd-na-hn & 180 & 2 & 7.1128 \\
h4-cc-na-hn & 180 & 2 & 7.1128 \\
\hline$E_{\mathrm{LJ}}\left(r_{i j}\right)=$ & $4 \varepsilon_{i j}\left\{\left(\frac{\sigma_{i j}}{r_{i j}}\right)^{12}\right.$ & $\left.-\left(\frac{\sigma_{i j}}{r_{i j}}\right)^{6}\right\}$ \\
\hline$E_{\mathrm{LJ}}$ & $\varepsilon_{i i}(\mathrm{~kJ} / \mathrm{mol})$ & $\sigma_{i i}(\mathrm{~nm})$ \\
\hline $\mathrm{na}$ & 0.8543730 & 0.3205810 \\
$\mathrm{nd}$ & 0.3937140 & 0.3384170 \\
$\mathrm{cc}$ & 0.4133790 & 0.3315210 \\
$\mathrm{~cd}$ & 0.4133790 & 0.3315210 \\
$\mathrm{hn}$ & 0.0418400 & 0.1106500 \\
$\mathrm{~h} 5$ & 0.0673624 & 0.2447300 \\
$\mathrm{~h} 4$ & 0.0673624 & 0.2536390 \\
\hline
\end{tabular}

